\title{
Evaluation of SIMESON, A Training Program to Improve Access to Quality Health Care for Pregnant Women and Newborn in Different Health Care Facilities of Northern Bangladesh
}

\section{Rina Das}

International Centre for Diarrhoeal Disease Research Bangladesh

\section{Fahmida Dil Farzana}

International Centre for Diarrhoeal Disease Research Bangladesh

\section{Zakia Sultana}

Terre des Hommes Foundation

Kallol Mukherji

Terre des Hommes Foundation

\section{Adnan Baayo}

Terre des Hommes Foundation

\section{Marufa Sultana}

International Centre for Diarrhoeal Disease Research Bangladesh

Nausad Ali

Bangladesh Institute of Development Studies

Mohammod Jobayer Chisti ( $\nabla$ chisti@icddrb.org )

International Centre for Diarrhoeal Disease Research Bangladesh

Shafiqul Alam Sarker

International Centre for Diarrhoeal Disease Research Bangladesh

\section{Tahmeed Ahmed}

International Centre for Diarrhoeal Disease Research Bangladesh

\section{ASG Faruque}

International Centre for Diarrhoeal Disease Research Bangladesh

\section{Research article}

Keywords: Evaluation, Perinatal health, PPH, SIMESON, Helping baby breathe, Bag and mask ventilation

Posted Date: August 25th, 2020

DOI: https://doi.org/10.21203/rs.3.rs-58898/v1 
License: (c) (i) This work is licensed under a Creative Commons Attribution 4.0 International License. Read Full License

Version of Record: A version of this preprint was published at Nursing Open on November 16th, 2021. See the published version at https://doi.org/10.1002/nop2.1131. 


\section{Abstract}

Background: Access to perinatal health care is improving in Bangladesh, but maternal and neonatal mortality is still a public health concern. SIMESON (simulation for essential skills for obstetrical and neonatal care) training was focused on expanding maternal-newborn health services for safe delivery by improving infrastructure, perinatal knowledge, and skills of health care providers working in health care facilities in northern Bangladesh. Our objective was to explore the current knowledge and skills of health care providers already trained by SIMESON, in reviewing perinatal health situation including the current status of health care facilities, cost estimation and most importantly delivering perinatal health services.

Methods: It was an observational study involving 88 health care providers working in 20 health care facilities. Cost estimation following both quantitative and qualitative approaches was also attempted.

Result: Knowledge and skills of health care providers regarding the provision of normal delivery and immediate postpartum care, management of postpartum hemorrhage (PPH), and retained placenta; and use of the bag and mask ventilation to help a baby breathe were found to be considerably strengthened following SIMESON training. During the 6 months after training, there were 477 PPH cases managed successfully with only 1 death; neonatal deaths observed were 6.6 per 1000 live births; twice the number of facility deliveries; and $80 \%$ use rate of Ambu bag for helping baby breathe. The estimated cost per trainee was 395.68 USD, and 5.85 USD per beneficiary.

Conclusion: SIMESON training improved maternal and child health by facilitating the health care professionals' improved knowledge and skills, enhancing access to health care facilities, and increasing facility delivery. Such a training program should be promoted within health system in hard-to-reach areas of the country that are burdened with other commitments.

\section{Background}

Access to maternal health care is improving in Bangladesh, maternal deaths have declined significantly, but the maternal mortality rate is still a public health concern (196 per 100,000 live births in 2016) [1]. Nearly half of these deaths are due to postpartum hemorrhage (PPH) and eclampsia. These two causes are still playing a significant role in maternal deaths (rates remained unchanged between 2010 and 2016 in Bangladesh) [2]. Newborn deaths are alarming and half of these deaths occur during the very first day of life, while $86 \%$ of newborn deaths occur within their first week of life [3]. Many of these deaths can be prevented by providing training to the health workers and developing their skills on some key issues related to perinatal care [4]. The percentage of births attended by trained health professionals has increased from $27 \%$ in 2010 to $50 \%$ in 2016 [5]. Moreover, pregnant women seeking institutional care for delivery have increased from $23 \%$ in 2010 to $47 \%$ in 2016 [6]. Treatment seeking for pregnancy-related complications has increased from 29\% in 2010 to $46 \%$ in 2016 [7]. Despite all these advancements, maternal and neonatal deaths are still enormously high. 
SIMESON (SIM for Simulation, ESON for Essential Skills for Obstetrical and Neonatal care) has been developed to address the needs of delivery service providers, particularly working in remote areas with limited access to facilities. SIMESON relies on low-dose high-frequency approach (facility-based learning modules have been developed to improve competency and confidence of the health care team, thus, to save more lives during pregnancy and at birth). It demonstrated impressive improvements in the identification and management of PPH, newborn asphyxia, and care of low birth weight baby [8]. Terre des hommes foundation (Tdh) implemented SIMESON in Mali to reduce stillbirths as well as neonatal and maternal mortality [9]. This training protocol has been successfully piloted in Mali and is currently aiming at scaling up of these encouraging achievements to other settings with unacceptably high rates of maternal and neonatal mortality. Through a total of 186 training sessions, overall skills improved by $45 \%$, health facilities improved their competencies by $45 \%$, and knowledge expanded by $90 \%$. Impressive improvements were noted in the identification and management of PPH, newborn asphyxia, and low birth weight baby care. Kurigram District is situated in North-East Bangladesh. It is a highly disaster-prone area and is known to be affected annually by heavy rains, waterlogging, flash flood, river erosion, etc. Data obtained from Kurigram Civil Surgeon office which is under Ministry of Health and Family Welfare (MOHFW) showed that in 2015 maternal mortality rate was 140 per 100,000 live births, half of them were due to $\mathrm{PPH}$, the neonatal mortality rate was 28.2 per 1,000 live births, and death due to birth asphyxia was $28.4 \%$. As a part of government and non-government efforts to tackle this situation, in 2019 Tdh implemented SIMESON in Kurigram aiming to increase the number of facility-based births conducted by qualified health personnel for expanding services to a larger number of women and newborn babies.

Here we aimed to measure the current knowledge and skills of SIMESON trained health care providers in delivering perinatal health services, assess the perinatal heath situation and readiness of health care facilities for the management of maternal and neonatal emergencies, and estimate the cost of SIMESON in Kurigram.

\section{Methods}

\section{Study design}

It was an observational investigation. The study followed both quantitative and qualitative approaches among health care providers who received SIMESON training.

\section{Study sites and study population}

The study was carried out in January 2020 in a total of 20 health care facilities (Kurigram 250 bedded Sadar Hospital, 8 Upazila Health Complexes, 8 Health and Family Welfare Centres, 1 Mother and Child Welfare Centre, and 2 Community Clinics) in Kurigram, a northern district of Bangladesh. The health care facilities were selected based on accessibility to delivery facilities, availability of staff trained in SIMESON, and provision of perinatal health services in a running infrastructure. Eighty-eight government health care providers (57 senior staff nurses, 14 midwives, 12 family welfare visitors, 3 family welfare 
assistants, and 2 community health care providers) who received SIMESON training and currently working in those facilities were assessed.

\section{Data collection}

Health care staff members, working at different government health facilities were the study participants who were selected through purposive sampling for observation of their perinatal care deliveries. A crosssectional design was followed to collect quantitative data. Data were collected using paper-based standardized questionnaires that have been validated by the Tdh team. The questionnaires were based on an internationally accepted protocol on Helping Mothers Survive (HMS) and Helping Babies Survive (HBS). Guidelines, checklists, and questionnaires were developed based on Tdh's baseline report and our evaluation proposal as well as the literature review. Informed consent was taken from each participant in a written format. Data collection process included (i) review of hospital logs, observation of the place of care for delivery and surroundings, (ii) administration of self-administered multiple-choice questionnaire (MCQ) to the trained health care providers, (iii) observation of their birthing practices, management of PPH and retained placenta and helping baby breathe, (iv) in-depth interviews of the participants, pregnant mothers, and their caregivers, who recently received perinatal care, key personnel of the facility, community leaders, local qualified health care providers, and nurses, and (v) financial record review to collect information on project implementation and monitoring related costs.

\section{Intervention}

\section{SIMESON training program}

The SIMESON project uses a mobile unit with two health workers specifically trained in medical simulation who make regular visits to each peripheral health facility to provide practical training in essential procedures. The overall aims of the project are to save the lives of mothers and their babies at birth in four domains: knowledge about routine care of post-partum for the mother, helping baby breathe [10], essential care for every baby [11, 12], and management of bleeding after birth [13, 14]. Additionally, the program involved specialized technologies (e.g. mannequins and video recordings) that allowed trainees to imagine a scenario that imitates the real-life setting without affecting patient safety [15].

An initial assessment of knowledge and skills was conducted in February 2019 to identify the training needs of staff members of these facilities. For knowledge 151 and for skills 148 government health care providers in 53 health care facilities of Kurigram were assessed.

Tdh used a collaborative approach to adapt the SIMESON and team-training program to make it culturally and contextually applicable to local settings of Kurigram. The training started in April 2019 with 148 health care providers from 32 intervention facilities. Among them, 128 participants completed the 6 months training. The training was led by eight trainers who conducted exercises, awareness assessments, and team-training events during the training. Over the intervention period, trainers provided 
monthly refresher training and monitored the performance, lasting about two to three hours, at each facility.

\section{Evaluation}

\section{i) Knowledge and skill assessment}

To examine the current knowledge status, quality of service delivery, scopes for improvements, and any likely scale-up, we measured the impact of training on providers' knowledge through a self-administered questionnaire, which providers filled out immediately before the commencement of the skill assessment during final evaluation. Health care providers who participated voluntarily were asked to answer a set of questions or statements. The knowledge assessment contained 33 questions of multiple choice on core topics that were responded by the participants. The skill assessment had 65 statements in which participants were required to demonstrate the SIMESON step by step in a mannequin. We were, however, able to collect this data from 88 (68.75\%) for knowledge assessment and $86(67.2 \%)$ for skill assessment out of the 128 providers during the evaluation (others could not be reached at the time of evaluation).

\section{ii) Facility assessment}

Of the 32 health care facilities, having the SIMESON training program we visited 20 facilities during the evaluation. We included perinatal health information, antenatal care (ANC) and postnatal care (PNC) information, number of deliveries, newborn health information, essential resources required: communication services, health registers/logs, and basic protocols, delivery room essential requirements/medical equipment and supplies, essential drugs required, and information on access to health care facilities.

\section{iii) Qualitative research}

We interviewed health care providers and pregnant women at the intervention facilities. For this, we conducted in-depth interviews (IDIs), key informant interviews (KIIs), and informal interviews for assessing challenges and barriers to the delivery of high-quality care and their familiarity with SIMESON training. The qualitative research was conducted with a purposive sample through 14 IDIs, 6 KIIs, and 3 informal interviews who participated voluntarily after giving written informed consent. IDIs were conducted among senior staff nurses, midwives, family welfare visitors, family welfare assistants, sub assistant community medical officers, and community health care providers. Guidelines were used to reveal information of interest and to allow interviewees to express their opinions and ideas in their own words. Interviews were undertaken until the saturation point was reached, i.e. no new information generated with further interviewing. Six KIls were conducted, and the key informants were elected representatives of the people of the locality, nursing supervisor, and monitoring officer of Tdh. Interviews for both IDIs and KIls lasted for approximately 30 minutes. Three informal interviews of hospitalized pregnant women after childbirth were conducted for assessing satisfaction levels for the services and 
obstetric care provided by health care facilities. Each of these interviews lasted for approximately 10 to 15 minutes. The verbatim was recorded on tape recorders and transcribed concurrently.

iv) Cost estimation:

Through the SIMESON training program perspective, economic costs were calculated for the analytical period between February and December 2019. Program expenditures data were collected from SIMESON's financial statements, expenses were divided into capital expenditure and operating expenditure. Interviews were conducted with Tdh's main program staff to understand the specific plan in phases and to identify the categories of costs by each of the activities. These costs associated with the SIMESON program were listed and estimated accordingly. Expenditures for staff included expenses for project team members. Materials and equipment included costs of the services needed for the project whereas the costs for facilities covered the cost of using some non-organization-owned workspaces. Other costs included recurrent cost items such as rent, utilities, printing, IT (information technology) installation, IT bills, cell phone, phone bills, stationery, janitorial, housekeeping supplies, leaflet, brochure, announcement materials, facilities for food, lodging, transportation, the recreation of participants, and remuneration for the faculties. All costs are expressed in CHF (Swiss Franc) with an exchange rate of 1 CHF $=1.03$ USD (US Dollar) for January 2020.

\section{Data quality and survey supervision}

Data collectors were properly oriented and trained on the tools before data collection. Overall data quality and survey were supervised by the investigators of icddr,b. Local-level coordination was facilitated by additional staff members of the Kurigram Tdh team. Data quality was checked randomly by the investigators through on -the -spot monitoring.

\section{Data analysis}

Data entry was done by the data management team of icddr,b who was not involved in data collection. Data cleaning, updating, range check, duplication check, consistency check, frequency check, and crosstabulation were performed by two separate data management assistants who were also involved in crosschecks. Raw data were rechecked if there were any discrepancies. Data were entered into SPSS, and descriptive statistics such as frequency distribution, percentage, and tabulations were performed as part of data analysis. We focused on descriptive statistics with quantitative data and, utilized a framework approach (widely known as thematic analysis or qualitative content analysis) with both the combined strategies of deductive and inductive types of analysis to deal with the qualitative data [16].

\section{Results}

\section{Knowledge assessment: comparison between baseline and evaluation}

After a comparison between baseline and evaluation findings, we found that (i) knowledge about normal vaginal delivery and routine postpartum care increased regarding- washing hands before normal delivery 
even before wearing the gloves ( $10 \%$ to $98.9 \%$ ), provision of family planning advice according to the need of the couple ( $10 \%$ to $88.6 \%$ ), and current recommendation on performing episiotomy (37\% to $72.7 \%$ ); (ii) newborn care: knowledge about helping babies to survive increased in consequence of suctioning unnecessarily or frequently ( $9 \%$ to $64.4 \%$ ) and in which area to be observed to diagnose severe jaundice in newborn (47\% to $67.8 \%$ ); (iii) maternal complications: knowledge about bleeding after completion of birth increased especially for the cervical tear (19\% to $62.1 \%)$ and manual removal of placenta $(26 \%$ to 62.1) (Table 1).

Table 1. Knowledge assessment: comparison between baseline and evaluation

Knowledge assessment
Baseline

(\%)

$(n=151)$
Evaluation (\%) $(n=88)$

$$
(n=151)
$$

\section{(i) Normal delivery and routine care in post-partum for the mother and helping babies survive}

\begin{tabular}{|c|c|c|}
\hline Washing hand for the delivery even if wearing gloves & $15(10)$ & $87(98.9)$ \\
\hline Current recommendation on performing an episiotomy & $56(37)$ & $64(72.7)$ \\
\hline Correct chronology of AMTSL & $49(33)$ & $54(61.4)$ \\
\hline $\begin{array}{l}\text { Provision of family planning: advice according to the needs of } \\
\text { the couple }\end{array}$ & $15(10)$ & $78(88.6)$ \\
\hline Time of measuring the newborn's heart rate & $16(24)$ & $82(94.3)$ \\
\hline Consequences of suctioning a baby unnecessarily & $13(9)$ & $56(64.4)$ \\
\hline
\end{tabular}

\section{(ii) Essential care for Every Baby}

Initiation of breastfeeding within the first hour after birth $99(66)$ $72(82.8)$ Normal temperature range $96(64)$ $72(82.8)$

Which area to be observed to diagnose severe jaundice in newborn

$71(47)$ $59(67.8)$

\section{(iii) Bleeding after Birth Complete}

\begin{tabular}{lll}
\hline Postpartum care after bimanual compression & $92(61)$ & $63(72.4)$ \\
\hline Management of PPH due to atony & $54(36)$ & $57(65.5)$ \\
\hline Management of PPH if uterotonics failed & $23(15)$ & $36(41.4)$ \\
\hline Initiation of manual removal of placenta & $13(9)$ & $43(49.4)$ \\
\hline Circumstances for manual removal of the placenta & $39(26)$ & $54(62.1)$ \\
\hline Medications are given before manual removal of placenta & $34(23)$ & $53(60.9)$ \\
\hline Check cervical lacerations & $29(19)$ & $54(62.1)$
\end{tabular}




\section{Skill assessment: comparison between baseline and evaluation}

a) Skills regarding normal delivery and routine care in immediate post-partum for the baby: Skills regarding normal delivery and routine care in immediate post-partum for the mother increased to $100 \%$ in case of almost all components and $86.3 \%$ in case of proper perineal wash. During the care of the newborn, the percentage of the newborn who had temperature measured soon after birth got reduced ( $42 \%$ to $34.9 \%$ ), counting the respiratory rate for one full minute was slightly increased (36\% to $38.4 \%$ ), skills increased in observed level of alertness and muscle tone (26\% to $77.9 \%)$, and weight measurement (56\% to $88.4 \%$ ). Practice to initiate breastfeeding within the first hour of birth and keeping the baby in mothers' arm, not with other relatives were both raised to $100 \%$ from $55 \%$ and $43 \%$ respectively (Table 2 ).

Table 2. Skills regarding routine care in immediate post-partum for the baby: comparison between baseline and evaluation

\begin{tabular}{|lll|}
\hline Skill assessment & \multicolumn{1}{|c|}{$\begin{array}{l}\text { Baseline (\%) } \\
(\mathrm{n}=148)\end{array}$} & $\begin{array}{l}\text { Evaluation (\%) } \\
(\mathrm{n}=86)\end{array}$ \\
\hline Routine care in immediate post-partum for the baby & \\
\hline Alertness and muscle tone & $39(26)$ & $67(77.9)$ \\
\hline Measure the temperature & $62(42)$ & $30(34.9)$ \\
\hline Count respiratory rate for a full minute & $53(36)$ & $33(38.4)$ \\
\hline Weight measurement of the newborn & $83(56)$ & $76(88.4)$ \\
\hline Examine the baby in the mother's arm & $64(43)$ & $86(100)$ \\
\hline Breastfeeding and bonding & $82(55)$ & $86(100)$ \\
\hline
\end{tabular}

b) Skills regarding - atony and post-partum hemorrhage: increased in looking for bleeding (66\% to $100 \%$ ), checking bladder and catheterizing the woman (31\% to $70 \%)$, measuring pulse and blood pressure, and initiating IV infusion along with oxytocin administration (47\% to $74.4 \%$ ), bimanual compression technique ( $9 \%$ to $76.7 \%$ ), rechecking of the uterus (37\% to $95.5 \%$ ), massaging of the uterus, and the practice of respectful maternity care (39\% to $100 \%$ ) (Fig 1).

Fig 1. Skills regarding- atony and post-partum hemorrhage: comparison between baseline and evaluation

c) Skills regarding - retained placenta and manual removal of the placenta: Increased in repeating $10 \mathrm{IU}$ IM oxytocin, encouraging emptying of the bladder, giving diazepam $10 \mathrm{mg} \mathrm{IM}$, and starting IV line with 
normal saline to prevent shock. Administering IV antibiotics, giving oxytocin $20 \mathrm{IU}$ in $1 \mathrm{~L}$ saline if the placenta is not removed, an examination of the placenta, checking uterine tone and its massaging, removal of the placenta, and controlled cord traction for each contraction (Fig 2).

\section{Fig 2. Skills regarding- retained placenta and manual removal of the placenta: comparison between baseline and Evaluation}

d) Skills regarding - bag and mask ventilation: All the participants correctly demonstrated the steps related to bag and mask ventilation during the evaluation, except the step of ventilating at 40 breaths per minute (range is $30-50 /$ minute) was reported from $37 \%$ to $83.7 \%$.

e) Skills regarding helping baby breathe: Increased in evaluating newborns' chest movement, effective ventilation, ventilation with bag and mask within the golden minute, close observation of the baby, recognition of the breathing in baby and normal heart rate, continuous ventilation, squeezing of the bag harder, and removal of secretions from the mouth and nose raised. Skills related to the removal of wet clothes reduced from $87 \%$ to $67.4 \%$. (Fig 3 ).

\section{Fig 3. Skills regarding helping baby breathe: comparison between baseline and evaluation}

\section{Facility assessment}

During the baseline assessment, the number of deliveries in 59 health care facilities was 2973 in 6 months which almost doubled during the evaluation, where a quarter of the deliveries were performed by the caesarian section. About 478 mothers experienced PPH. There were 2 maternal deaths, among them one was due to PPH and another one was as a result of eclampsia. About $43.9 \% \mathrm{PPH}$ was due to cervical or vaginal tear, $26 \%$ from prolonged labor, and $20 \%$ as a result of retained placenta. Other causes included uterine atony, retention of products of conception, placenta previa, maternal infection, and maternal comorbidities (e.g. maternal high blood pressure, diabetes mellitus, etc.). Neonatal death was 6.6 per 1000 live births, among them, eight newborn died due to low birth weight and birth asphyxia.

All the facilities had soap/alcohol hand rub for hand hygiene, $95 \%$ facilities had a functional delivery table, $55 \%$ facilities had 3 colored waste bin, $70 \%$ facilities reported presence of baby scale, and $80 \%$ facilities had Ambu bag, only $45 \%$ facilities mentioned having baby length board. Eighty-five percent of facilities had oral antibiotics and $40 \%$ indicated availability of the IV antibiotics for mothers, $75 \%$ of facilities were maintaining oxytocin supply, and $65 \%$ of facilities had IV fluid supply.

All facilities visited during evaluation indicated that no fees were required for normal delivery and newborn care, $70 \%$ facilities had clear referral criteria, $40 \%$ indicated the presence of complaints box or register/log and those facilities had accountability mechanisms for addressing the mistreatments or violations, and $85 \%$ facilities had informed consent procedure.

\section{Findings from qualitative research}


The qualitative data highlighted the expectations of providers about the training and its effect on their knowledge and skill. Representative quotations from IDIs, KIls, and informal interviews are thematically shown here.

\section{Theme 1. The overall perception of SIMESON training}

Participants appreciated the experience immensely and were thankful for getting the opportunity to be included in the training. Some of them stated that the training had refreshed their knowledge of essential skills while others suggested including some new topics in the training curriculum. Participants expected an improvement in their skills due to this training and desired more training opportunities like this. They also acknowledged improvements in their competence and confidence during the management of obstetric and neonatal emergencies, as well as coordination and collaboration between patient-providers.

"... I learned a lot from Tdh (SIMESON training) ... and now if I face such kinds of emergencies, I can manage that... I believe SIMESON training helped me a lot."

\section{Theme 2. The objective of SIMESON training}

The respondents understood that the main objective of the SIMESON training was to reduce maternal and neonatal mortality. Few more information were obtained from interviews, for example, the number of institutional deliveries increased, ideal labor rooms being set-up, quality of maternal and newborn care increased, hygiene during normal vaginal delivery better maintained as of late, application of PPH management techniques (e.g. bimanual compression) and helping baby breathe skill acquired. "..... the objective was to increase the practice of normal delivery in Health Care Facility.... sometimes, home delivery causes complications...... that's why they (Tdh) targeted to increase the number of facility delivery..... and eventually reduce maternal and neonatal mortality"

\section{Theme 3. Effectiveness of SIMESON training}

The participants mentioned that the SIMESON training helped them to improve their skills regarding normal vaginal delivery, which were reflected through their comments -

"There are certain instructions like what we should do during normal delivery, who would be the in-charge of what etc. in detail. This (the SIMESON training) has significantly improved my skills..." and "This training helped us in remembering the things that we need to apply during the management of normal vaginal delivery of a pregnant woman."

Theme 4. Benefits of the training

It was refreshers training for most of the participants.

"Tdh (SIMESON) served as a supplementary training, which helped me to overcome my shortcomings in conducting normal delivery......" 
Regarding the benefits of the pregnant women, the participants quoted, "Women died previously due to lack of skill in managing PPH......" and "The women are happy now, they said that they would recommend others to go for normal delivery in the health facility"

Regarding the benefits of the newborn, participants mentioned, "We used to be very nervous during baby resuscitation, now we can handle it quite well" and "Now we use the penguin sucker; we have learned how to help a baby breathe......."

Theme 5. Experience of the participants after the training

Tdh team successfully improved the skills of healthcare personnel in normal vaginal delivery, the participants have become more aware of hygiene, the whole process is more organized and systematic now, the confidence of participants has increased significantly, they have learned new techniques like bimanual compression, wearing gloves, etc., PPH management is currently done more efficiently.

"If we see excessive bleeding in PPH, right away we apply bi-manual compression to manage the case."

\section{Theme 6. Challenges faced}

Due to the shifting duty hours, there was limited availability of the participants during the training period. It was difficult to organize all the participants in a prefixed time due to their heavy workload. The participants found it difficult to engage themselves in the training with full concentration when it was arranged during their duty hours. Lack of understanding of the patients' attendants about the use of the bag and mask for the resuscitation of the neonate and reluctance of others to disclose their pregnancy status and to attend the ANC in due time were other challenges encountered.

\section{Theme 7. The satisfaction of the participants}

Participants were doing their tasks more efficiently in recent days. This was a hands-on training that helped the participants to retain their skills aptly. The duration of any maneuver related to normal vaginal delivery, baby resuscitation, and management of PPH has significantly reduced, and the processes themselves have become much easier to perform. Regarding respectful maternity care, one participant said, "...... from the training we also learned to express empathy towards the mother and the baby......"

\section{Theme 8. Suggestions for improvement}

Participants were asked about what they might recommend for enhancing the quality of training. Most of them recommended extending the training period, including more sessions, and regular conduction of such training.

"The training had a short duration, ended rather quickly, if more time were allocated for the training, we would have learned better". 
Some participants proposed to shorten the training hours but increase the training period. There was a strong consensus among attendees that more health professionals should be included to participate in the training to support facility-wide progress.

"The number of participants should be increased (a diverse group of staff); all the staff members (of a facility) need to know about these things ......."

Participants suggested to include more clinical topics like; management of eclampsia, how to repair a perineal tear, and Kangaroo mother care. Participants who are currently serving at the peripheral government health centers stated that they could not apply some of the skills acquired through the simulation training due to a lack of medical and obstetrical instruments.

"If the equipment can be arranged, it would be good to perform the procedures we have learned in the training...... we do not have sufficient instruments in the facility ....... some of the equipment has become old and rusty".

\section{Cost estimation}

The cost estimation of the SIMSON training program revealed costs of CHF 384.16 (395.68 USD) per trainee (health care provider) and CHF 5.68 (5.85 USD) per indirect beneficiary (mother and newborn) who sought care from facilities (Table 3 ).

Table 3: Cost estimation of SIMESON training program 


\begin{tabular}{|c|c|c|c|c|}
\hline $\begin{array}{l}\text { Cost } \\
\text { components }\end{array}$ & Inputs & $\mathrm{CHF}^{*}$ & USD & $\begin{array}{l}\% \text { of the } \\
\text { total } \\
\text { cost }\end{array}$ \\
\hline \multirow{6}{*}{$\begin{array}{l}\text { Capital } \\
\text { expenditures }\end{array}$} & National Coordinator Health & 867 & 893.01 & 1.76 \\
\hline & Project Officer & 1,350 & 1390.5 & 2.74 \\
\hline & Basic training & 9,491 & $9,775.73$ & 17.4 \\
\hline & Capital (i.e. laptop) & 261 & 268.83 & 0.5 \\
\hline & BCC (i.e., poster, leaflet) & 6,967 & $7,176.01$ & 12.8 \\
\hline & Staff/personnel (i.e., staff nurses) & 30,278 & $31,186.34$ & 55.4 \\
\hline \multicolumn{2}{|c|}{ Total Capital expenditures } & 49,214 & $50,690.42$ & 86.0 \\
\hline \multirow{5}{*}{$\begin{array}{l}\text { Operating } \\
\text { expenditures }\end{array}$} & Regular training & 5,030 & $5,180.9$ & 8.8 \\
\hline & Stationeries & 165 & 169.95 & 0.3 \\
\hline & Meeting & 345 & 355.35 & 0.6 \\
\hline & Social mobilization (i.e. Communications) & 753 & 775.59 & 1.3 \\
\hline & $\begin{array}{l}\text { Conveyance for service provider (i.e. local } \\
\text { transport, rent car) }\end{array}$ & 1,348 & $1,388.44$ & 2.4 \\
\hline \multicolumn{2}{|c|}{ Total Operating expenditures } & 7,641 & $7,870.23$ & 13.4 \\
\hline \multicolumn{2}{|l|}{ Total cost } & 56,855 & $58,560.65$ & 100.0 \\
\hline \multicolumn{2}{|c|}{ Cost per direct beneficiary $(n=148)$} & 384.16 & 395.68 & \\
\hline \multicolumn{2}{|c|}{ Cost per indirect beneficiary $(n=10000)$} & 5.68 & 5.85 & \\
\hline
\end{tabular}

\section{Discussion}

This is one of the few studies that specifically combined both knowledge and skills for quality of care to minimize maternal and neonatal mortality in a single intervention and, to our knowledge, the first to use SIMESON as the training tool in Bangladesh. Here, we found that SIMESON training has expanded the knowledge and skills of health care providers in treating obstetric and neonatal emergencies. Moreover, the perceptions of the health care providers suggest that this type of training is very much helpful in their capacity building and they are eagerly looking forward to the continuation of the training for a longer period in the larger population. By visualizing the facility assessment results, we can comprehend that the training resulted in improvements as regards the number of facility delivery and decreased maternal and neonatal mortality.

Health care providers who took part in a simulation-based training course on obstetric emergencies of a tertiary care university hospital of the United Kingdom also reported a noticeable improvement in both of 
their knowledge and skills when dealing with real-life emergencies observed in prospective follow-up research [17]. The incorporation of training with the "learning by doing" approach meant that professionals understood and practiced all the skills of emergency obstetric and neonatal emergency care (EmONC) and minimized the limited learning opportunities identified in prior EmONC training sessions [18].

After a comparison between baseline and evaluation, knowledge increased in washing hands before normal delivery, even before wearing the gloves, provision of family planning advice according to the need of the couple, current recommendation on performing an episiotomy, and where to look for cervical tear and the skill of manual removal of placenta. Regarding neonatal care knowledge also increased in understanding the adverse consequence of suctioning a newborn when performed unnecessarily, and which part of the baby to be observed to diagnose severe jaundice in case of a newborn. Similarly, a mixed-method study on integrated simulation training on emergency obstetric and neonatal care and respectful maternity care in Ghana observed that providers' knowledge increased from an average of $61.6 \%$ at pre-test to $74.5 \%$ at post-test [19].

During skill assessment, we found that participants were very prompt in conducting normal delivery. Researchers have reported that an intensive training session for vaginal deliveries on an obstetric mannequin or birth pelvis model at the beginning of the obstetrics clerkship automatically and substantially improved the self-assurance of medical students to engage in an uncomplicated, supervised attempt for vaginal deliveries in a teaching hospital of Canada [20]. A quasi-experimental study conducted in Pakistan reported significantly better post-training results after a simulation-based normal delivery skill learning [21]. Participants usually did not miss any steps. Data from several other simulation studies found that the application of deliberate practice to simulation training narrowed differences in performance in pediatric residents of USA and obstetric and neonatal emergencies of Northern Guatemala $[22,23]$.

Properly following the steps of PPH management procedure were also increased from baseline to evaluation. In Northern Tanzania, skills expanded from $19 \%$ before, to $43 \%$ immediately after, to $48 \%$ at nine months after training on bimanual uterine compression [14]. Similar work has shown that multidisciplinary in situ simulation activities enhance the management of obstetric emergencies such as $\mathrm{PPH}$ and is a safe and effective way to develop skills and improve health care processes [24]. The skills of the trainees on the management of retained placenta and manual removal of the placenta were highly improved in all the steps of the procedure from baseline. Studies have observed that trainees exhibited positive changes in attitude; as well as the perception of overall team performance in a simulated environment of obstetric emergency care [25]. Similar research studies have reported active training methods, such as simulations, case-based learning, hands-on skills training, and direct feedback reviews are components of successful in-service training to enhance health care providers' knowledge and skills in Cuernavaca, Mexico [26, 27]. 
All the participants correctly performed the steps regarding the bag and mask ventilation. Similar program assessments that took place in India and Kenya which were strongly acceptable, demonstrated increased confidence, enhanced knowledge, and acquired skills [11]. Performance regarding helping baby breathe also increased from baseline to evaluation. Skills related to the evaluation of chest movement, effective ventilation, and ventilation with bag and mask within the golden minute raised significantly. Participants in a program on helping babies breathe for neonatal resuscitation in resource-limited settings (Pakistan and Kenya) demonstrated high satisfaction, high self-efficacy, and advancement in knowledge and skills [28]. During the management of newborn babies immediately after birth, skills regarding the measurement of the newborns' temperature and removal of wet clothes were decreased from baseline, it was probably due to giving more emphasis on other steps like the bag and mask ventilation, eye medication, convulsion assessment, etc. while avoiding others by the participants.

According to Bangladesh Maternal Mortality and Health Care Survey 2016, the most common causes of maternal mortality were hemorrhage (31\%), followed by eclampsia, and abortion-related complications [1]. Hemorrhage and eclampsia accounted for 55\% of all maternal deaths in 2016 [1]. In our evaluation, we found two maternal deaths due to PPH and eclampsia which were lower than that of the national data. We found cervical and vaginal tears (45\%) were the main causes of PPH. However, the causes of $\mathrm{PPH}$ varied in different studies. Studies on associated factors of PPH in Pakistan reported that PPH is caused by cervical, vaginal and perineal tears occurring in $24 \%$ cases [29] and a similar study in Nigeria revealed cervical and vaginal tears occurring in $14 \%$ cases [30]. In Zimbabwe, a case-control study reported that cases were much more likely than controls to have a traumatic delivery involving vagina or cervix and causing tears, which accounted for more than one-third of the hemorrhage [31].

Bangladesh Demographic and Health Survey (BDHS) 2017-18 indicated a neonatal mortality rate of 30 per 1000 live births in 2015 in Bangladesh [32]. In our evaluation, we found neonatal deaths at 6.6 per 1000 live births. This observation represented the neonatal death among facility-based deliveries and did not include the number of neonatal deaths in the community, this might explain the reasonable lower number of neonatal deaths in the 20 health care facilities. Worldwide the major causes of neonatal mortality are low birth weight and birth asphyxia $[33,34]$ and we observed similar results during our evaluation. The number of institutional deliveries almost doubled from baseline to evaluation and according to BDHS 2017-2018, facility delivery has increased from $15 \%$ to $50 \%$ in Bangladesh in the last 10 years [32].

The results of the performance evaluation showed that providers appreciated the quality of the training which included both the information context and the skill content. The respondents understood that the main objective of the SIMESON training was to reduce maternal and neonatal mortality. Several studies showed that their intervention incorporated contents of deliberate training such as engaged learners, welldefined objectives, resident level complexity, and concentrated repeated training with direct input from professional trainers $[15,35]$. 
Participants were experiencing several issues during and after successful completion of the training such as improving the skills in managing normal delivery. They also had positive opinions about the training. They acknowledged changes in their expertise and trust in treating obstetric and neonatal crises, as well as coordination and collaboration between patient-provider. In a similar study in Ghana, more than 95\% of the participants reported that the training was beneficial for them and that they should use the skills acquired in their real-life [19]. Some of the participants mentioned that the exchange of greetings and respect as well as the provision of continued support to the pregnant women who are in labor should be an essential part of respectful maternity care that they learned from the training. Different research results indicate that comprehensive training that provides healthcare providers with the ability to understand, practice, and comment on their delivery of supportive maternity care in the sense of intense emergency obstetric simulations has the potential to increase the childbirth experience of women in lowresource settings [36].

After experiencing several challenges and difficulties, participants still have satisfaction regarding the SIMESON training; this was a hands-on training that helped the participants to retain skill aptly. Similar changes have been identified in the skills of pediatric and internal medicine residents after several deliberate practice sessions over longer periods in Tripler Army Medical Center, Honolulu, and the internal medicine residency program at Northwestern University, Chicago respectively [37, 38]. The duration of any maneuver regarding normal delivery, baby resuscitation, and management of PPH has reduced, and the process has become much easier.

Increasing the knowledge and skill of health care providers by implementing the SIMESON training program is the most effective and single intervention with reasonable costs for reducing maternal and neonatal mortality. SIMESON training program is a low-cost program in a rural area of Bangladesh. Although we have done the cost estimation of SIMESON, we were not able to compare our results with other programs due to a lack of similar studies with identical technicality, timeline, and geography. The cost per indirect beneficiary was considerably low, the estimated cost per direct beneficiary (trainee health care providers) was reasonable.

Both qualitative and quantitative approaches, relatively large sample for quantitative study design, diversity in participants to capture maximum variability by the qualitative research design, site visits to facilitate real-life observations were the strengths of the evaluation. The evaluation had some limitations. Shorter time available for evaluation during clinical rotations of the participants limited enough opportunities for knowledge and skill assessment. We were unable to assess actual (real-life) improvements in the skill of obstetric and neonatal emergencies and knowledge retention as the practices were mainly observed in the mannequin except for a few normal deliveries with essential care of newborn babies in a real-life situation at facilities. Advanced analyses were not done due to a lack of access to baseline data by the evaluation team. Finally, convenience sampling was used for qualitative research. So, findings from the qualitative data may not be generalizable outside the resource-poor settings. Our results indicate that this SIMESON training is practical, appropriate, and efficient in resource-limited environments in improving maternal and child health in Bangladesh. 


\section{Conclusion}

Post-training skills of health care providers strengthened in all four components of maternal and newborn care. Through this training, there were improvements in the quality of care for maternal and neonatal health services at the government health care facilities of Kurigram. Taking up such training may guide efforts to provide healthy, sensitive, respectful, high quality obstetric and neonatal care even in remote rural settings of a larger population in Bangladesh. As most of the skills and knowledge increased from baseline, we recommend the SIMESON training to continue with regular monitoring and periodic evaluation. To improve the quality of subsequent training, the causes of knowledge and skill reduction in some components should be explored. Incorporating the management of eclampsia, and suturing skill component in the training to manage perineal tear will further reduce maternal morbidity and mortality. Kangaroo mother care component should be introduced to reduce neonatal mortality. However, sustainability is depending on implementing the training with similar materials and methodologies by the Bangladesh Government as one of the components of regular refresher training in expanded areas.

\section{Abbreviations}

AMTSL Active Management of the Third Stage of Labor

\begin{tabular}{ll}
\hline ANC & Antenatal care \\
\hline EmONC & Emergency Obstetric and Neonatal Care \\
\hline HBB & Helping Baby Breathe \\
\hline HCFs & Health care Facilities \\
\hline icddr,b & International Centre for Diarrhoeal Disease Research, Bangladesh \\
\hline IDI & In-depth interview \\
\hline KII & Key informant interview \\
\hline PNC & Postnatal care \\
\hline PPH & Post-partum hemorrhage \\
\hline SIMESON & Simulation for Essential Skills in Obstetrical and Neonatal care \\
\hline Tdh & Terre des hommes foundation \\
\hline
\end{tabular}

\section{Declarations}

\section{Ethics approval and consent to participate}

Tdh has a memorandum of understanding (MoU) with the Ministry of Health and Family Welfare (MoHFW) in Bangladesh. Under this MoU, Tdh is responsible for conducting the formal assessment and evaluation in collaboration with the family planning authorities. The study was initiated after getting 
approval from the Institutional Review Board of icddr,b which is comprised of the Research Review Committee and Ethical Review Committee. Signed written informed consent was obtained from the participants. The respondents had the freedom to withdraw themselves from participation at any point in the study. The consent form was read out to the participant if he/she was unable to read (particularly the women of the locality). The confidentiality of the participants was maintained strictly.

\section{Consent for publication}

Not applicable.

\section{Availability of data and materials}

Due to concerns about participants' confidentiality, data is available on request. The full dataset has not been stored in a public repository as it contains participants' names, contact numbers, institution names and a few identifiers that may lead to the identification of individual participants. However, the identification of participants is not possible from the data presented in the article as it contains summarized results only. The full dataset is freely available upon written request to the corresponding author. Request for the study dataset may be sent to Dr. Mohammod Jobayer Chisti, Senior Scientist, icddr,b. Email: chisti@icddrb.org.

\section{Competing interests}

The authors declare no conflict of interest regarding the publication of this paper.

\section{Funding}

This study was financially supported by Tdh. The funding body had no role in the design of the study, data collection, analysis, interpretation of the findings.

\section{Authors' contributions}

Tdh conceived and developed the SIMESON training program. Tdh and icddr,b evaluation team developed the data collection tools. RD managed the data set and analyzed the data, developed the tables/graphs, and wrote the initial draft of the manuscript. RD and FDF developed the qualitative research guideline and FDF wrote the result for the qualitative part. MS and NA analyzed the cost estimation part. SAS, MJC, TA, and ASGF critically reviewed the manuscript and gave intellectual inputs. All authors contributed to the final version of the paper.

\section{Acknowledgments}

We acknowledge with gratitude the commitment of the SIMESON program as supported by Tdh. The authors are grateful to Tdh Kurigram staff, trained government health care providers of Kurigram, mothers, and children from Kurigram for their invaluable contributions. We acknowledge the contribution of icddr,b's core donors including Government of the People's Republic of Bangladesh, Global Affairs 
Canada, Canada; Swedish International Development Cooperation Agency and the Department for International Development, UK Aid for their continuous support and commitment to the icddr,b's research efforts.

\section{References}

1. National Institute of Population Research Training, International Centre for Diarrhoeal Disease Research, Bangladesh, and MEASURE Evaluation, Bangladesh Maternal Mortality and Health Care Survey 2016: Preliminary Report. 2017.

2. Shah, R., Q. Nahar, and E.S. Gurley, One in Five Maternal Deaths in Bangladesh Associated with Acute Jaundice: Results from a National Maternal Mortality Survey. The American journal of tropical medicine and hygiene, 2016. 94(3): p. 695-697.

3. Horton, R. and U. Samarasekera, Stillbirths: ending an epidemic of grief. Lancet, 2016. 387(10018): p. 515-516.

4. Ariff, S., et al., Evaluation of health workforce competence in maternal and neonatal issues in public health sector of Pakistan: an Assessment of their training needs. BMC Health Services Research, 2010. 10(1): p. 319.

5. Kibria, G.M.A., et al., A comparison of practices, distributions and determinants of birth attendance in two divisions with highest and lowest skilled delivery attendance in Bangladesh. BMC pregnancy and childbirth, 2018. 18(1): p. 122-122.

6. Siddique, A.B., et al., Antenatal care in rural Bangladesh: Gaps in adequate coverage and content. PLOS ONE, 2018. 13(11): p. e0205149.

7. Filippi, V., et al., Levels and causes of maternal morbidity and mortality. Disease control priorities, 2016. 2.

8. Nelissen, E., et al., Clinical performance and patient outcome after simulation-based training in prevention and management of postpartum haemorrhage: An educational intervention study in a low-resource setting. BMC Pregnancy and Childbirth, 2017. 17.

9. Terre des hommes foundation, Acting together because every child counts: Annual report 2014 - Terre des hommes. 2015, Terre des hommes. p. 9.

10. Chaudhury, S., et al., Cost analysis of large-scale implementation of the 'Helping Babies Breathe' newborn resuscitation-training program in Tanzania. BMC health services research, 2016. 16(1): $p$. 681-681.

11. Thukral, A., et al., Evaluation of an educational program for essential newborn care in resourcelimited settings: Essential Care for Every Baby. BMC pediatrics, 2015. 15: p. 71-71.

12. World Health Organization, et al., Pregnancy, childbirth, postpartum and newborn care: a guide for essential practice. 3rd ed. ed. 2015, Geneva: World Health Organization.

13. Evans, C.L., et al., Competency-based training "Helping Mothers Survive: Bleeding after Birth" for providers from central and remote facilities in three countries. International Journal of Gynecology \& 
Obstetrics, 2014. 126(3): p. 286-290.

14. Nelissen, E., et al., Helping Mothers Survive Bleeding After Birth: retention of knowledge, skills, and confidence nine months after obstetric simulation-based training. BMC pregnancy and childbirth, 2015. 15: p. 190-190.

15. Campbell, D.M., et al., High-fidelity simulation in neonatal resuscitation. Paediatrics \& child health, 2009. 14(1): p. 19-23.

16. Gale, N.K., et al., Using the framework method for the analysis of qualitative data in multi-disciplinary health research. BMC medical research methodology, 2013. 13(1): p. 1-8.

17. Reynolds, A., D. Ayres-de-Campos, and M. Lobo, Self-perceived impact of simulation-based training on the management of real-life obstetrical emergencies. Eur J Obstet Gynecol Reprod Biol, 2011. 159(1): p. 72-6.

18. Gueye, M., et al., Simulation training for emergency obstetric and neonatal care in Senegal preliminary results. Med Sante Trop, 2017. 27(2): p. 131-134.

19. Afulani, P.A., et al., Provider knowledge and perceptions following an integrated simulation training on emergency obstetric and neonatal care and respectful maternity care: A mixed-methods study in Ghana. Midwifery, 2020. 85: p. 102667.

20. Sabourin, J.N., et al., Confidence in performing normal vaginal delivery in the obstetrics clerkship: a randomized trial of two simulators. J Obstet Gynaecol Can, 2014. 36(7): p. 620-627.

21. Shah, N., et al., Simulation based medical education; teaching normal delivery on intermediate fidelity simulator to medical students. J Pak Med Assoc, 2017. 67(10): p. 1476-1481.

22. Cordero, L., et al., Deliberate practice improves pediatric residents' skills and team behaviors during simulated neonatal resuscitation. Clinical pediatrics, 2013. 52(8): p. 747-752.

23. Fahey, J.O., et al., Promoting cultural humility during labor and birth: putting theory into action during PRONTO obstetric and neonatal emergency training. The Journal of Perinatal \& Neonatal Nursing, 2013. 27(1): p. 36-42.

24. Lutgendorf, M.A., et al., Multidisciplinary In Situ Simulation-Based Training as a Postpartum Hemorrhage Quality Improvement Project. Mil Med, 2017. 182(3): p. e1762-e1766.

25. Robertson, B., et al., Simulation-based crisis team training for multidisciplinary obstetric providers. Simul Healthc, 2009. 4(2): p. 77-83.

26. Cohen, S.R., et al., Self-efficacy change with low-tech, high-fidelity obstetric simulation training for midwives and nurses in Mexico. Clinical Simulation in Nursing, 2012. 8(1): p. e15-e24.

27. Bluestone, J., et al., Effective in-service training techniques, timing, setting and media: evidence from an integrative review of the literature. Baltimore: Jhpiego Corporation, 2012.

28. Singhal, N., et al., Helping Babies Breathe: global neonatal resuscitation program development and formative educational evaluation. Resuscitation, 2012. 83(1): p. 90-6.

29. Naz, H., et al., Maternal morbidity and mortality due to primary PPH-experience at Ayub Teaching Hospital Abbottabad. J Ayub Med Coll Abbottabad, 2008. 20(2): p. 59-65. 
30. Selo-Ojeme, D.O. and F.E. Okonofua, Risk factors for primary postpartum haemorrhage. A case control study. Arch Gynecol Obstet, 1997. 259(4): p. 179-87.

31. Tsu, V.D., Postpartum haemorrhage in Zimbabwe: a risk factor analysis. Br J Obstet Gynaecol, 1993. 100(4): p. 327-33.

32. Zaman, R., et al., Joint UNICEF Bangladesh-Government of Bangladesh Health Programme Mid-Term Evaluation 2017-2020. 2019.

33. Bahl, R., et al., Setting research priorities to reduce global mortality from preterm birth and low birth weight by 2015. Journal of global health, 2012. 2(1).

34. de Almeida, M.F.B., et al., Early neonatal deaths with perinatal asphyxia in very low birth weight Brazilian infants. Journal of Perinatology, 2015. 35(11): p. 954-957.

35. McGaghie, W.C., et al., Does simulation-based medical education with deliberate practice yield better results than traditional clinical education? A meta-analytic comparative review of the evidence. Academic medicine: journal of the Association of American Medical Colleges, 2011. 86(6): p. 706.

36. Afulani, P.A., et al., Can an integrated obstetric emergency simulation training improve respectful maternity care? Results from a pilot study in Ghana. Birth, 2019. 46(3): p. 523-532.

37. Sawyer, T., et al., Deliberate practice using simulation improves neonatal resuscitation performance. Simulation in Healthcare, 2011. 6(6): p. 327-336.

38. Wayne, D.B., et al., Mastery learning of advanced cardiac life support skills by internal medicine residents using simulation technology and deliberate practice. Journal of general internal medicine, 2006. 21(3): p. 251-256.

\section{Figures}

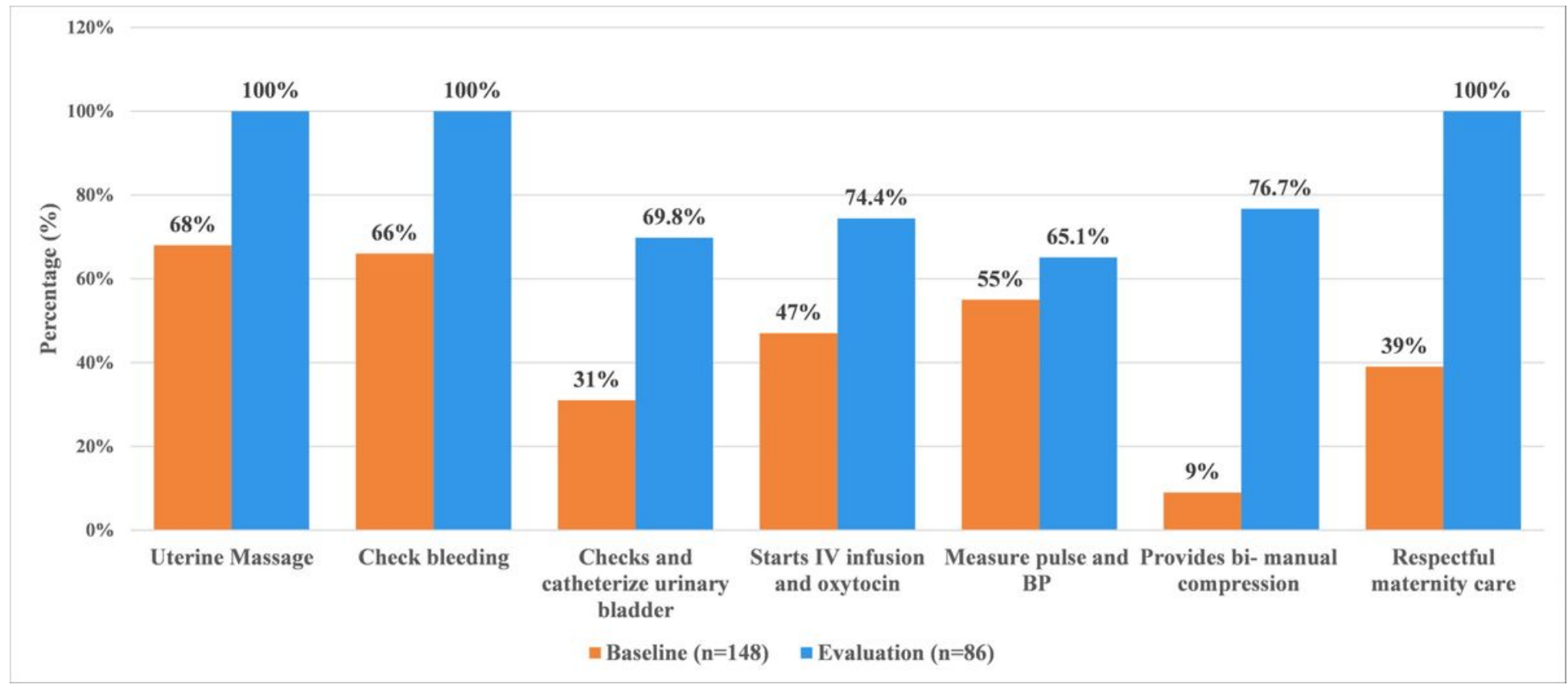


Skills regarding - atony and post-partum hemorrhage: comparison between baseline and evaluation

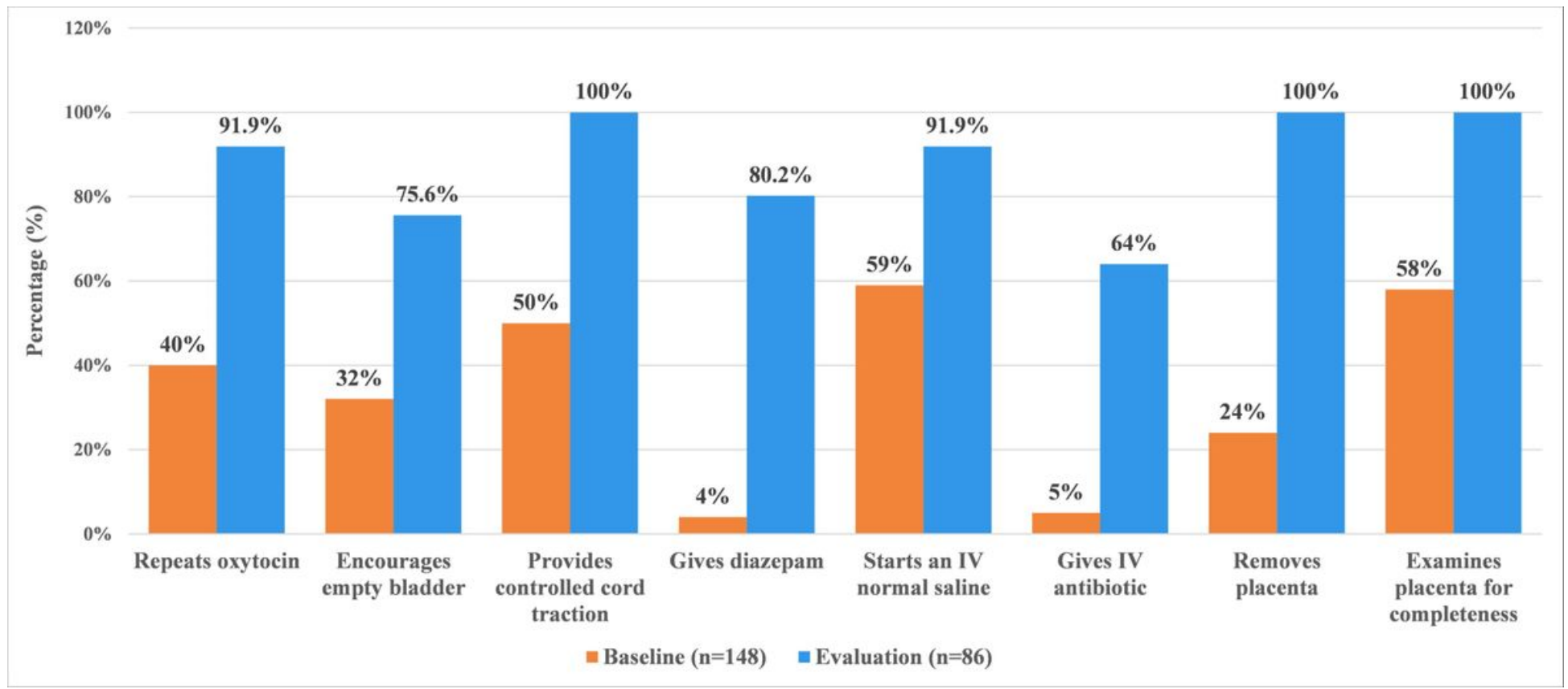

Figure 2

Skills regarding - retained placenta and manual removal of the placenta: comparison between baseline and Evaluation

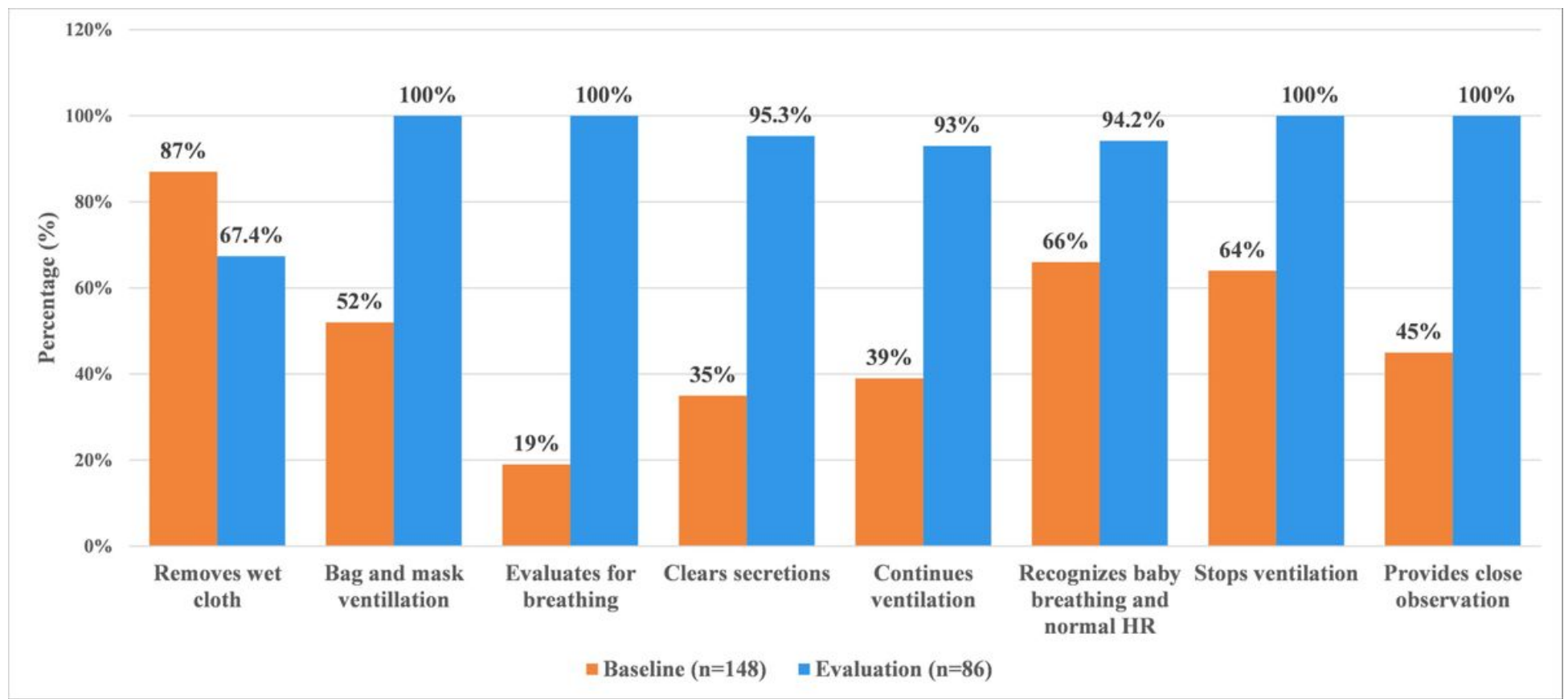

\section{Figure 3}

Skills regarding helping baby breathe: comparison between baseline and evaluation

\section{Supplementary Files}


This is a list of supplementary files associated with this preprint. Click to download.

- SIMESONskillassessmentchecklist.docx

- SIMESONquestionnaire.docx 